\title{
Scan Blindness Phenomenon in Conformal Finite Phased Arrays of Printed Dipoles
}

\author{
Vakur B. Ertürk, Member, IEEE, Onur Bakır, Student Member, IEEE, Roberto G. Rojas, Fellow, IEEE, and
} Baris Güner

\begin{abstract}
Scan blindness phenomenon for finite phased arrays of printed dipoles on material coated, electrically large circular cylinders is investigated. Effects on the scan blindness mechanism of several array and supporting structure parameters, including curvature effects, are observed and discussed. A full-wave solution, based on a hybrid method of moments/Green's function technique in the spatial domain, is used to achieve the aforementioned goals. Numerical results show that the curvature affects the surface waves and hence the mutual coupling between array elements. As a result, the array current distribution of arrays mounted on coated cylinders are considerably different compared to similar arrays on planar platforms. Therefore, finite phased arrays of printed dipoles on coated cylinders show different behavior in terms of scan blindness phenomenon compared to their planar counterparts. Furthermore, this phenomenon is completely different for axially and circumferentially oriented printed dipoles on coated cylinders suggesting that particular element types might be important for cylindrical arrays.
\end{abstract}

Index Terms-Coated cylinders, conformal arrays, Green's function, method of moments (MoM), scan blindness.

\section{INTRODUCTION}

A RRAYS of printed antenna elements have been successfully implemented in the past for beam scanning and other applications [1], [2]. Therefore, several design tools and numerical techniques have been developed and implemented in CAD packages for the design and analysis of planar printed finite and infinite arrays [3]-[11]. Lately, many commercial (e.g., mobile base stations, transmitters and receivers for multi-input multi-output (MIMO) systems), military (e.g., airborne, missile borne arrays) as well as some biomedical applications require phased arrays that conform to curved host platforms. This is mainly due to aerodynamic constraints, reduced radar cross section, wider scan range (compared to arrays on planar platforms) and aesthetic reasons. Such arrays (planar or curved) might have many elements on dielectric substrates (or in free space), where electromagnetic coupling through space and surface waves can lead to scan blindness [3] and seriously degrade the performance of a system. This phenomenon was once addressed as a "catastrophic effect" by Schaubert et al. [12]. Therefore, a complete

Manuscript received December 25, 2004; revised December 12, 2005. This work was supported in part by the Turkish Scientific and Technological Research Agency (TÜBİTAK) under Grant EEEAG-104E044.

V. B. Ertürk and O. Bakır are with the Department of Electrical and Electronics Engineering, Bilkent University, TR-06800 Bilkent, Ankara, Turkey (e-mail: vakur@ee.bilkent.edu.tr).

R. G. Rojas and B. Güner are with the Department of Electrical and Computer Engineering, ElectroScience Laboratory, The Ohio-State University, Columbus, OH 43212-1191 USA.

Digital Object Identifier 10.1109/TAP.2006.875482 understanding of the scan blindness phenomenon is required to improve the scan range of phased arrays and to reduce design costs significantly.

The blindness phenomenon, which was defined (for planar infinite arrays of printed antennas) as a phase matching between the phase progression of a surface wave $\left(\beta_{\mathrm{sw}}\right)$ on the dielectric substrate and the phase progression of a certain Floquet mode ([3], [13]), has been previously investigated in detail for various infinite and finite arrays of printed antennas on grounded planar dielectric substrates. The blindness mechanism was carefully explained first for infinite arrays of printed antennas [3], [4], [14], and then research on this topic was extended to finite phased arrays of printed antennas [5], [6]. Later, this phenomenon was discussed for different array configurations such as infinite array of monopoles in a grounded dielectric slab [15], infinite arrays of printed dipoles on dielectric sheets perpendicular to a ground plane [16], infinite stripline-fed tapered slot antenna arrays with a ground plane [12], [17]. Furthermore, various methods to improve the scan range such as subarraying [13], substrate modification [18], loading the array elements with varactor diodes [19] or using shorting posts [20] were reported. However, the common point in all these aforementioned studies is the fact that arrays (infinite or finite) are mounted on planar platforms. To the best of our knowledge, no similar investigations has been presented for arrays of printed elements mounted on dielectric coated curved surfaces, where the curvature of the supporting structure affects the blindness mechanism as well as various performance metrics of the array.

Therefore, in this paper, scan blindness phenomenon is investigated for several arrays consisting of finite number of axially and/or circumferentially oriented printed dipoles on various-sized electrically large, dielectric coated, circular cylinders with different electrical parameters. Effects of several array and supporting structure parameters on the scan blindness mechanism as well as on various characteristics of arrays are observed. Furthermore, a one-to-one comparison between arrays of printed dipoles on aforementioned cylinders and arrays of printed dipoles on grounded planar dielectric slabs is made in terms of the blindness phenomenon. It is shown that the orientation of the array elements combined with the curvature effects play an important role on the behavior of the surface waves, which in turn can alter the scan blindness in these structures. To achieve these goals, a hybrid method of moments $(\mathrm{MoM}) /$ Green's function technique in the spatial domain is used [21]-[23]. This method is basically an element-by-element approach in which the mutual coupling between dipoles through space and surface waves is incorporated. It has been 

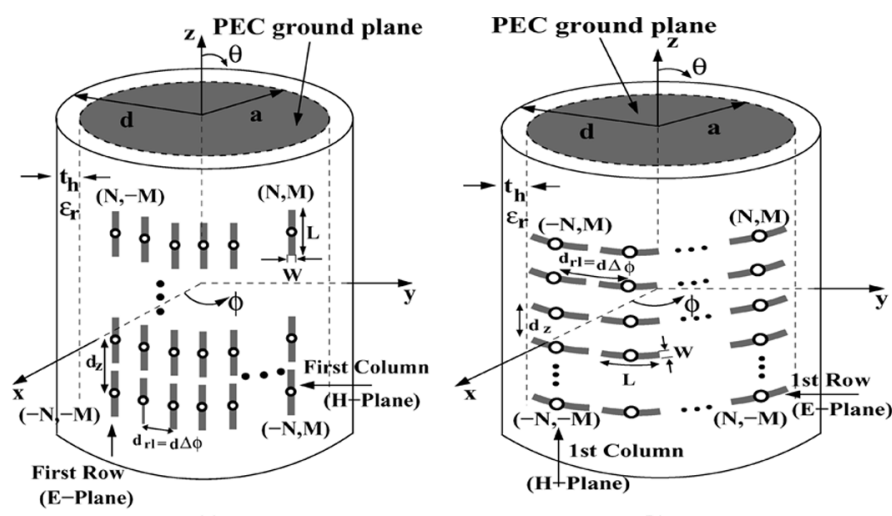

(b)

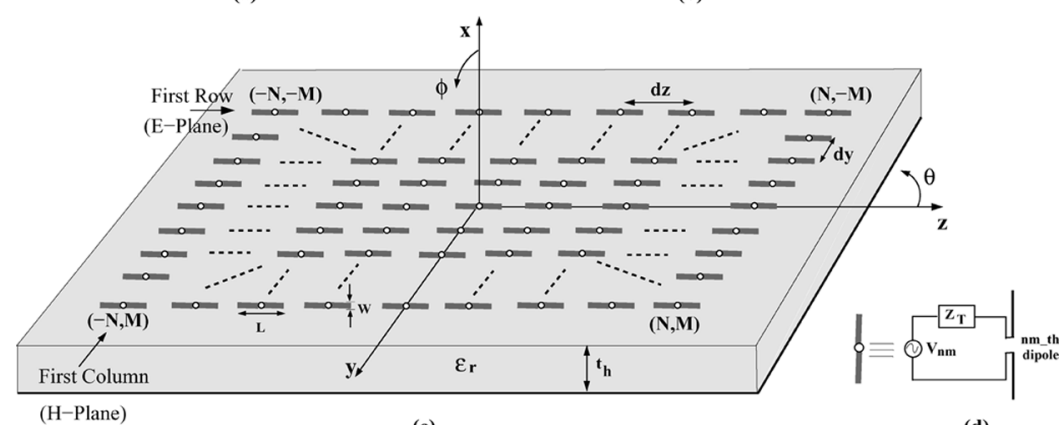

(c)

(d)

Fig. 1. Geometries of periodic arrays of $(2 N+1) \times(2 M+1)$ (a) axially and (b) circumferentially oriented printed dipoles on dielectric coated, electrically large circular cylinders. (c) Geometry of a periodic, planar array of $(2 N+1) \times(2 M+1)$ printed dipoles. (d) Dipole connected to an infinitesimal generator with a voltage $V_{n m}$ and a terminating impedance $Z_{T}$.

recently used for the full-wave analysis of both axially and circumferentially oriented printed dipoles on electrically large, material coated, circular cylinders, and very accurate results have been obtained for all cases [22], [23].

In Section II, the geometry and the formulation of the problem are presented. Several numerical examples are given in Section III to demonstrate the effects of the curvature of the host body (coated cylinder) on the surface waves and blindness mechanism. The importance of the array element orientation with respect to the curvature of the host body is discussed. Furthermore, how several electrical and geometrical parameters of the array together with its supporting structure affect the basic performance metrics of finite arrays of printed dipoles on coated cylinders are investigated. An $e^{j \omega t}$ time dependence is assumed and suppressed throughout this paper.

\section{FORMULATION}

\section{A. Geometry}

Fig. 1(a) and (b) show the geometries of finite, periodic arrays of $(2 N+1) \times(2 M+1)$ axially $(\hat{z}$-directed) and circumferentially ( $\hat{\phi}$-directed) oriented printed dipoles, respectively. The arrays are mounted on the dielectric-air interface of dielectric coated, perfectly conducting, circular cylinders, which are assumed to be infinitely long along the $z$-direction. The coated cylinders have an inner radius denoted by $a$, outer radius denoted by $d$, and hence the coating thickness $t_{h}=d-a$. Finally, the relative permittivity of the coating is $\epsilon_{r}>1$. For comparison purposes, the geometry of a finite, planar, periodic array of
$(2 N+1) \times(2 M+1)$ printed dipoles is also given in Fig. 1(c). In all three geometries, the dipoles are assumed to be center-fed with infinitesimal generators with impedance $Z_{T}$ as depicted in Fig. 1(d). Each dipole has a length $L$, width $W$, and is uniformly spaced from its neighbors by distances $d_{r l}=d \Delta \phi$ and $d_{z}$ in the $r l$ - $(r l=d \phi)$ and $z$-directions, respectively. Similarly for the planar case, each dipole is uniformly spaced from its neighbors by distances $d_{y}$ and $d_{z}$ in the $y$ - and $z$-directions, respectively.

\section{B. The Full-Wave Solution}

The full-wave solution used in this paper is a hybrid MoM/ Green's function technique in the spatial domain as explained in detail in [21]-[23]. Briefly, an electric field integral equation (EFIE) is formed, and applying a Galerkin MoM approach the following matrix equation is obtained [5], [22], [23]:

$$
\left([\mathbf{Z}]+\left[\mathbf{Z}_{\mathbf{T}}\right]\right) \cdot \mathbf{I}=\mathbf{V} .
$$

In the course of obtaining (1), dipoles are assumed to be thin ( $W \ll L)$ and a single expansion mode is used to represent the current on each dipole.

In (1), $[\mathbf{Z}]=\left[Z_{p q, n m}\right]$ is the impedance matrix of the array with elements $Z_{p q, n m}$, which denotes the mutual impedance between the $n m$ th and $p q$ th $(-N \leq n, p \leq N,-M \leq m, q \leq$ $M)$ dipoles, $\left[\mathbf{Z}_{\mathbf{T}}\right]$ is the generator terminating impedance matrix which is diagonal [5], $\mathbf{I}=\left[I_{n m}\right]$ is the unknown vector of expansion coefficients, and finally $\mathbf{V}$, given by

$$
\mathbf{V}=\left[V_{p q}=V_{0} e^{-j k_{0} d \cos (\phi-p \Delta \phi) \sin \theta} e^{-j k_{0} q d_{z} \cos \theta}\right]
$$


denotes the excitation of the $p q$ th dipole, where an ideal delta gap generator at the terminal of each center-fed dipole is assumed. Note that $(\theta, \phi)$ in (2) is the scan direction of the main beam, and $V_{0}=1$ for uniform excitations similar to [5], [22], [23]. Furthermore, the Toeplitz property of the matrix $[\mathbf{Z}]$ is employed to reduce the computational time and LU-decomposition method is applied in the solution of the matrix equation given by (1).

Because of the large number of printed dipoles mounted on these large coated circular cylinders, special attention must be given to the efficient calculation of $Z_{p q, n m}$ given by

$$
Z_{p q, n m}=\int_{S_{p q}} d s_{p q} \int_{S_{n m}} d s_{n m}^{\prime} f_{p q}\left(\mathbf{r}_{p q}\right) G_{u u}\left(\mathbf{r}_{p q} / \mathbf{r}_{n m}^{\prime}\right) f_{n m}\left(\mathbf{r}_{n m}^{\prime}\right) .
$$

In (3), $f_{n m}\left(\mathbf{r}_{n m}^{\prime}\right)$ and $f_{p q}\left(\mathbf{r}_{p q}\right)$ are the piecewise sinusoidal basis and testing functions with $\mathbf{r}_{p q}$ and $\mathbf{r}_{n m}^{\prime}$ being the position vectors of the $p q$ th and $n m$ th dipoles, respectively. The computational efficiency as well as the accuracy of this method are strongly dependent on the calculation of the appropriate dyadic Green's function component $G_{u u}\left(\mathbf{r}_{p q} / \mathbf{r}_{n m}^{\prime}\right)(u=z$ or $\phi$, depending on the orientation of the dipole) for arbitrary source and observation locations. Therefore, three different spatial-domain Green's function representations ([24]-[26]), each accurate and computationally very efficient in a given region of space, are used in conjunction with a switching algorithm so that $Z_{p q, n m}$ can be evaluated accurately and efficiently for arbitrary $n m$ th and $p q$ th dipole locations. These three Green's function representations are as follows: i) The planar representation which is valid when the field is evaluated in the vicinity of the source (i.e. valid in the source region). It is used based on the assumption that for electrically large ( $d$ is large) material coated circular cylinders and small separations $(s)$, the surface can be treated as locally flat. Hence, an efficient integral representation of the planar microstrip dyadic Green's function [24] is used for the self term evaluations of the impedance matrix. ii) The steepest descent path (SDP) representation of the dyadic Green's function [25], which is used away from both the paraxial (nearly axial) and the source regions (see [22]). This representation tends to become more efficient and accurate as the separation between the source and field points increases. iii) The paraxial spatial domain representation of the dyadic Green's function [26], which is used along the paraxial region of the array of both $\hat{z}$ and $\hat{\phi}$-directed printed dipoles, as well as in an annular-like region located around the source region of the array of $\hat{\phi}$-directed printed dipoles. This representation is derived to complement the SDP representation along the paraxial region. However, the $G_{\phi \phi}\left(\mathbf{r}_{p q} / \mathbf{r}_{n m}^{\prime}\right)$ component can be made valid away from the paraxial region by performing a slight modification to its curvature correction term [27]. Therefore, it is slightly more accurate than the SDP representation in the annular-like region around the source region, though its accuracy and efficiency become worse (compared to the SDP representation) for large separations other than the paraxial region.

The efficiency and accuracy of these Green's function representations, in particular the SDP and the paraxial representations, have been discussed previously in [21]-[26]. However, for

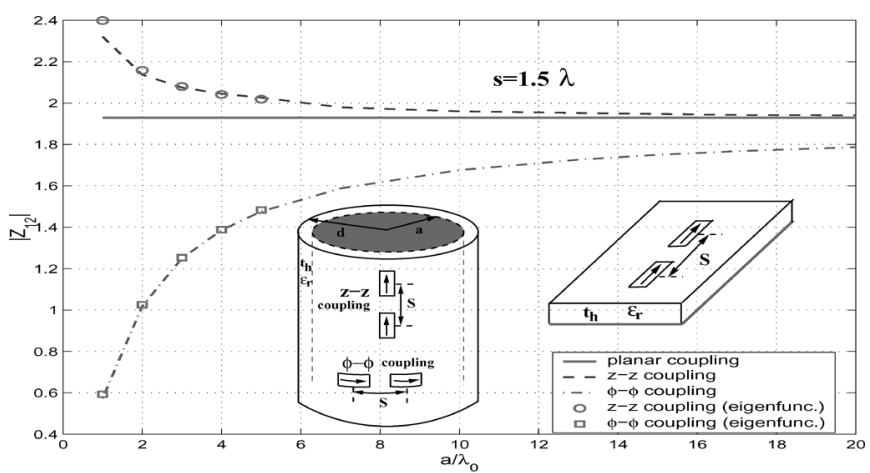

(a)

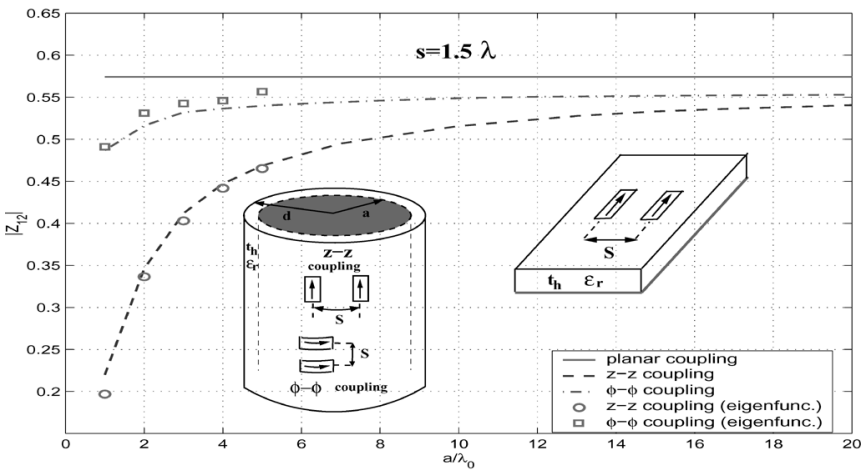

(b)

Fig. 2. Magnitude of the mutual coupling $\left|Z_{12}\right|$, between two identical $\hat{z}$-directed and $\hat{\phi}$-directed current modes versus inner radius $a$ evaluated at $s=$ $1.5 \lambda_{0}$ for $t_{h}=0.06 \lambda_{0}$ and $\epsilon_{r}=3.25$ along the (a) E-plane and (b) H-plane. The size of the current modes is: $(L, W)=\left(0.39 \lambda_{0}, 0.01 \lambda_{0}\right)$.

the sake of completeness it is worthwhile to briefly discuss their limitations in this paper. These limitations are manifested in the electrical size (i.e., the radius) of the coated cylinder and/or in the thickness of the coating. Note that the dielectric constant of the coating can always be linked to the thickness. First of all, the SDP and the paraxial representations are developed for electrically large coated cylinders. Therefore, the desired accuracy is generally achieved when the radius is greater than $1 \lambda_{0}$ ( $\lambda_{0}$ : free space wavelength). This is illustrated in Fig. 2, where the mutual coupling between two identical $\hat{z}$-directed and $\hat{\phi}$-directed current modes are plotted as a function of the inner radius $a$, and compared with the eigenfunction solution (spectral domain solution). The current modes are selected to be $(L, W)=\left(0.39 \lambda_{0}, 0.01 \lambda_{0}\right)$, the thickness is chosen as $0.06 \lambda_{0}$ and the relative dielectric constant of the coating is set to 3.25 . The couplings are evaluated at $s=1.5 \lambda_{0}$. The eigenfunction solution is plotted up to $a=5 \lambda_{0}$ since it exhibits serious convergence problems for greater radii. As expected, Green's function representations show excellent agreement with the eigenfunction solution (even for $a=1 \lambda_{0}$ ). The small difference in the $\phi-\phi$ coupling in Fig. 2(b) (especially at $a=5 \lambda_{0}$ ) is due to the convergence problems of the eigenfunction solution. Furthermore, the results approach the planar case with increasing cylinder radius without exhibiting any problems. On the other hand, these Green's function representations loose their accuracy when the thickness and/or relative dielectric constant of the coating increase. This is due to certain approximations (Debye, Watson, Olver's Uniform approximations) made for the ratios 
of special functions as explained in [25] and [27]. For the desired accuracy, an approximate upper limit is defined in [27] such that the thickness of the coating must be less than $0.2 \lambda_{d}$, where $\lambda_{d}=\lambda_{0} / \sqrt{\epsilon_{r}}$.

Finally, these three Green's function representations are combined to span the whole cylinder surface using two slightly different switching algorithms for the arrays of $\hat{z}$ - and $\hat{\phi}$-directed printed dipoles. In both algorithms, the air-dielectric interface of the cylinder is divided into three regions and on each region, the corresponding aforementioned Green's function representation is used. For the array of $\hat{z}$-directed printed dipoles, the switching algorithm is given by

$$
\begin{aligned}
& G_{z z} \\
& \quad= \begin{cases}\text { Planar representation } & s<0.4 \lambda_{0} \\
& \text { (i.e self-term evaluations) } \\
\text { SDP representation } & \left(\xi_{\mathrm{SP}} / s>0.2\right) \\
& \bigcap\left(s \geq 0.4 \lambda_{0}\right) \\
\text { Paraxial representation } & \left(\xi_{\mathrm{SP}} / s \leq 0.2\right) \\
& \bigcap\left(s \geq 0.4 \lambda_{0}\right)\end{cases}
\end{aligned}
$$

which is similar to the switching algorithm used in [21], [22], previously. However, the switching algorithm used for the array of $\hat{\phi}$-directed printed dipoles is different than the switching algorithm given in [21] and [23], and can be expressed as in (5) at the bottom of the page. In both (4) and (5), $\xi_{\mathrm{SP}}$ is the saddle point value of $\xi$ which is a parameter used in the SDP representation, and is given by $\xi_{\mathrm{SP}}=\left(d k_{0} \cos \alpha / 2\right)^{1 / 3}\left(\phi-\phi^{\prime}\right)$ in [25] with $\alpha$ being the angle between the ray path and the circumferential axis. Furthermore, around each boundary which divides the regions defined in (4) and (5), more than one Green's function representation yield almost the same accuracy. Hence, small variations in boundary definitions do not significantly affect the overall accuracy. Consequently, in addition to its accuracy and efficiency, the method is also very robust.

\section{Other Definitions and Far-Field Patterns}

By obtaining the mode currents from the solution of matrix equation (1), several performance metrics for phased arrays given in [1], [2], [5] are calculated to investigate scan blindness phenomenon for various cylindrical arrays of printed dipoles. Furthermore, calculated results for these performance metrics are compared with those for planar arrays. Among these performance metrics, the input impedance at the $n m$ th dipole is computed as

$$
Z_{\mathrm{in}}^{n m}=\frac{V_{n m}}{I_{n m}}
$$

and is used in the calculation of the active reflection coefficient at the $n m$ th dipole given by

$$
R^{n m}(\theta, \phi)=\frac{Z_{\mathrm{in}}^{n m}(\theta, \phi)-Z_{\mathrm{in}}^{n m}\left(\theta=90^{\circ}, \phi=0^{\circ}\right)}{Z_{\mathrm{in}}^{n m}(\theta, \phi)+Z_{\mathrm{in}}^{n m^{*}}\left(\theta=90^{\circ}, \phi=0^{\circ}\right)} .
$$

By defining the active reflection coefficient at the $n m$ th dipole as in (7), each array element is conjugate matched to its broadside scan impedance. Note that in some calculations (e.g., to quantify the nonuniformity in the input impedance across the finite array) the active reflection coefficient definition given by (7) can be modified, and a fixed element's input impedance at broadside scan can be used as a reference. For instance, if the middle element is chosen as a reference element, then the modified version of (7) is given by

$$
R_{\text {mid }}^{n m}(\theta, \phi)=\frac{Z_{\mathrm{in}}^{n m}(\theta, \phi)-Z_{\mathrm{in}}^{\mathrm{mid}}\left(\theta=90^{\circ}, \phi=0^{\circ}\right)}{Z_{\mathrm{in}}^{n m}(\theta, \phi)+Z_{\mathrm{in}}^{\mathrm{mid} *}\left(\theta=90^{\circ}, \phi=0^{\circ}\right)}
$$

where the subscript/superscript "mid" stands for the middle element of the array.

Another important metric is the active element pattern $E^{n m}(\theta, \phi)$ (and hence, the active element gain), which is the field radiated by the array when the $n m$ th dipole is excited by a voltage generator, and all other dipoles are terminated in an impedance $Z_{T}$ [5]. As explained in [5], this pattern gives a very good estimate of the gain pattern of the array even for small finite ones. The active element pattern for the $n m$ th dipole is calculated by setting the feed voltage of this dipole to unity whereas feed voltages for all other dipoles are set to zero. The dipole currents are computed from the solution of (1) by setting $Z_{T}$ equal to the conjugate of the isolated dipole input impedance. Then the active element pattern for the $n m$ th dipole is calculated as

$$
\begin{aligned}
& E_{n m}(\theta, \phi)=E_{n m}^{d}(\theta, \phi) \sum_{p=-N}^{N} \sum_{q=-M}^{M} I_{p q} \\
& \times e^{-j k_{0} d \cos (\phi-p \Delta \phi) \sin \theta} e^{-j k_{0} q d_{z} \cos \theta}
\end{aligned}
$$

where $E_{n m}^{d}(\theta, \phi)$ is the far-field element pattern of a single dipole on a dielectric coated circular cylinder calculated either asymptotically as presented in [28] or using a reciprocity approach as presented in [29]. In both solutions, the dipole current coefficients $\left(I_{n m}\right)$ obtained from the solution of (1) are used,

$$
G_{\phi \phi}= \begin{cases}\text { Planar representation } & s<0.4 \lambda_{0} \text { (i.e, self-term evaluations) } \\ \text { SDP representation } & \left(\xi_{\mathrm{SP}} / s>0.2\right) \bigcap\left(s \geq 2 \lambda_{0}\right) \\ \text { Paraxial representation } & {\left[\left(\xi_{\mathrm{SP}} / s \leq 0.2\right) \bigcap\left(s \geq 0.4 \lambda_{0}\right) \text { or }\left(\xi_{\mathrm{SP}} / s>0.2\right) \bigcap\left(0.4 \lambda_{0} \leq s<2 \lambda_{0}\right)\right]}\end{cases}
$$


and both solutions yield exactly the same result. Once the active element pattern is determined, the active element gain of the $n m$ th element is calculated as

$$
G_{n m}(\theta, \phi)=\frac{4 \pi\left|E_{n m}(\theta, \phi)\right|^{2}}{Z_{0} P_{\text {in }}}
$$

where $P_{\text {in }}$ is the power delivered to the $n m$ th element given by

$$
P_{\text {in }}=R e\left\{\sum_{p=-N}^{N} \sum_{q=-M}^{M} I_{p q} Z_{p q, n m} I_{n m}^{*}\right\}
$$

and $Z_{0}=120 \pi$ is the free-space intrinsic impedance.

Finally, the majority of the numerical results for both cylindrical and planar arrays are given in the principle planes, namely, the E- and H-planes. Therefore, making use of Fig. 1(a)-(c), where $\theta$ and $\phi$ are defined from the $z$ - and $x$-axis, respectively, the E- and $\mathrm{H}$-planes are defined as follows. For the array of $\hat{z}$-directed printed dipoles on coated cylinders and array of printed dipoles on planar grounded dielectric slabs, as depicted in Fig. 1(a) and (c), respectively, E-plane is the $x z$ plane and H-plane is the $x y$ plane. Hence, to scan the E-plane $\phi$ is set to $0^{\circ}$ and $\theta$ is varied, whereas to scan the H-plane $\theta$ is set to $90^{\circ}$ and $\phi$ is varied. However, for the array of $\hat{\phi}$-directed printed dipoles on coated cylinders, as depicted in Fig. 1(b), E-plane is the $x y$ plane and H-plane is the $x z$ plane. Thus, to scan the E-plane $\theta$ is set to $90^{\circ}$ and $\phi$ is varied, whereas to scan the H-plane $\phi$ is set to $0^{\circ}$ and $\theta$ is varied.

\section{NUMERICAL RESULTS AND DISCUSSION}

Numerical results are presented: i) to demonstrate effects of the curvature combined with array element orientation on the surface waves and scan blindness mechanism; ii) to investigate effects of several electrical and geometrical parameters of arrays together with their host platforms on the aforementioned performance metrics. In all results presented in this paper, the size of each dipole is selected to be $(L, W)=\left(0.39 \lambda_{0}, 0.01 \lambda_{0}\right)$, the periodicity of arrays is chosen to be $0.5 \lambda_{0}$ (i.e. $d_{z}=d_{r l}=$ $d_{y}=0.5 \lambda_{0}$ ), and finally $\epsilon_{r}=3.25$ is used. Furthermore, all the cylindrical arrays are excited using the right-hand side of (2). A similar excitation is used for the planar arrays [5].

The numerical results depicted in Fig. 3(a) and Fig. 3(b) show the magnitude of the reflection coefficient $|R|$ [defined in (7)] versus scan angle in the $\mathrm{E}$ - and $\mathrm{H}$-planes, respectively. The arrays are $11 \times 11 \hat{z}$ - and $\hat{\phi}$-directed printed dipoles on a $3 \lambda_{0}$ coated cylinder with $t_{h}=0.06 \lambda_{0}$. These results are also compared with those of a planar array (of $\hat{z}$-directed dipoles) with the same parameters $\left(t_{h}\right.$, number of elements, etc.). The $|R|$ values of all the arrays are computed at their center elements, which are conjugate matched to broadside scan. A possible scan blindness is observed at $\theta=41^{\circ}\left(90^{\circ}-\theta=49^{\circ}\right)$ for the cylindrical array of $\hat{z}$-directed printed dipoles along the E-plane as shown in Fig. 3(a). At this angle, the reflection coefficient of the center element has a magnitude greater than unity $(|R|>1)$, which means that its input impedance has a negative real part (i.e., $\operatorname{Re}\left(Z_{\mathrm{in}}^{\mathrm{mid}}\right)<0$ ). Therefore, this dipole delivers power to its generator implying that this power is delivered from other

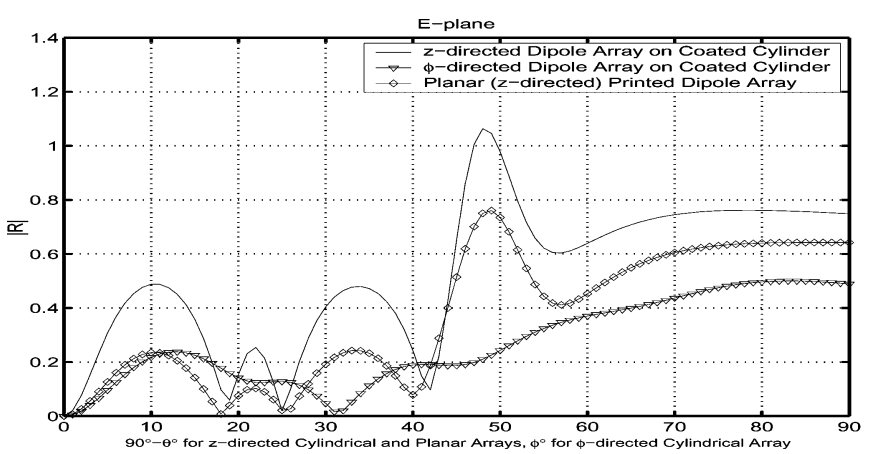

(a)

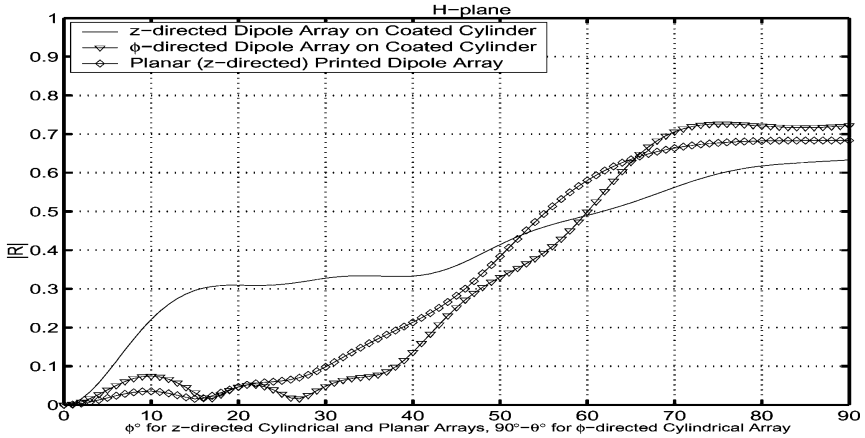

(b)

Fig. 3. Magnitude of the reflection coefficient, $|R|$, of the middle element versus scan angle comparison for $11 \times 11$ cylindrical arrays of axially $(\hat{z})$ and circumferentially $(\hat{\phi})$ directed printed dipoles, and the same array $(\hat{z}$-directed dipoles) on a planar grounded dielectric slab along the (a) E-plane and (b) $\mathrm{H}$-plane. Array and host body parameters are: $(L, W)=\left(0.39 \lambda_{0}, 0.01 \lambda_{0}\right)$, $\epsilon_{r}=3.25, t_{h}=0.06 \lambda_{0}, d_{z}=d_{r l}=d_{y}=0.5 \lambda_{0}, a=3 \lambda_{0}$.

ports with $|R|<1$ (i.e., $\operatorname{Re}\left(Z_{\mathrm{in}}^{n m}\right)>0$ ) to the middle element. Note that in finite arrays the $|R|>1$ condition for the center element of the array has been used as a tool to demonstrate the existence/possibility of scan blindness in [5], [7]. Thus, existence of this condition is also treated as an indication of a possible blindness in this paper. However, neither the array of $\hat{\phi}$-directed printed dipoles (on the same coated cylinder) nor the planar array shows blindness at this angle. Also it is observed that the shape of $|R|$ corresponding to the planar case is similar to that of the cylindrical array of $\hat{z}$-directed dipoles and it peaks around the same angle (but $|R|<1$ ). This may also suggest a potential scan blindness angle for the planar case. On the other hand, none of the arrays shows a scan blindness along the H-plane as illustrated in Fig. 3(b). This indicates that the E-plane is more critical for relatively thin coatings since only the lowest-order surface wave is present, which confines scan blindness phenomenon to the E-plane [30]. Since the blindness mechanism is closely related to the surface wave fields excited within the substrate of the arrays [3]-[5], the curvature of the supporting structure combined with the array element orientation will change the behavior of these fields. In particular, along the E-plane, surface waves of the $\hat{z}$-directed dipoles on coated cylinders are stronger than $\hat{\phi}$-directed ones and printed dipoles on planar grounded dielectric slabs [22], [23] [also see Fig. 2(a)]. Therefore, if the electrical and geometrical parameters of the array together with its host platform vary in a way to reinforce the surface waves, the possibility of observing a scan blindness increases, especially 


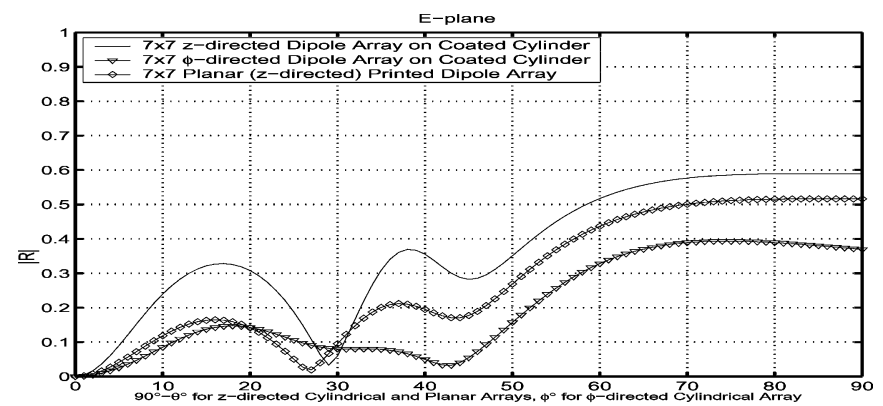

(a)

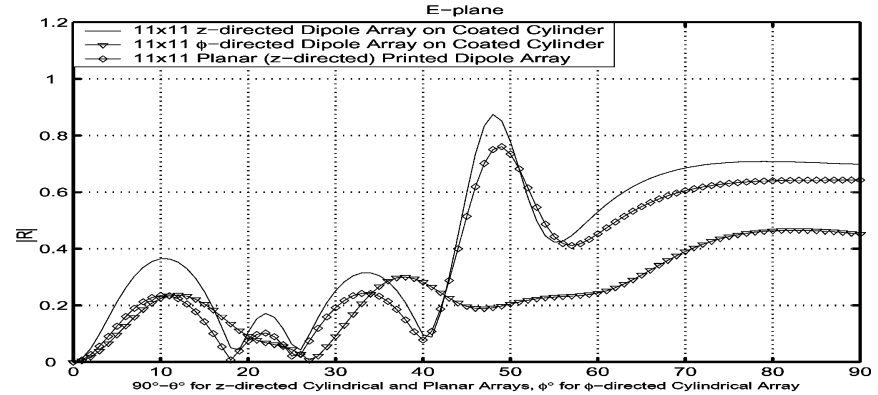

(b)

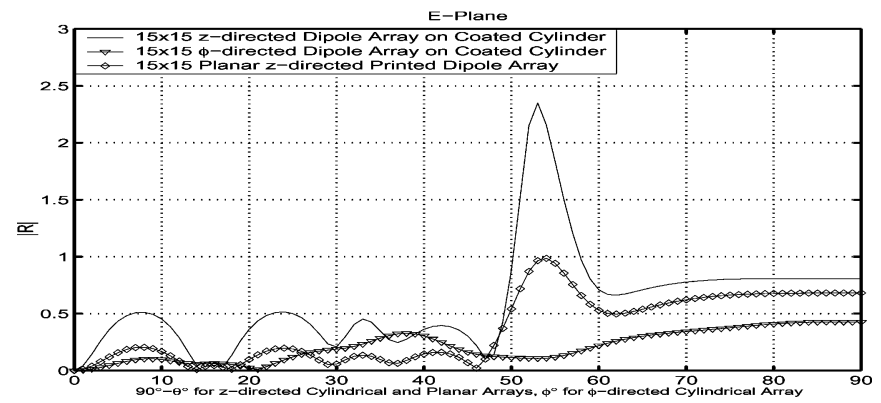

(c)

Fig. 4. Magnitude of the reflection coefficient, $|R|$, of the middle element versus scan angle along the E-plane for (a) $7 \times 7$, (b) $11 \times 11$, and (c) 15 $\times 15 \hat{z}$ - and $\hat{\phi}$-directed printed dipoles on a $4 \lambda_{0}$ coated cylinder. Planar array of $\hat{z}$-directed dipoles is also included. Other array and host body parameters are: $(L, W)=\left(0.39 \lambda_{0}, 0.01 \lambda_{0}\right), \epsilon_{r}=3.25, t_{h}=0.06 \lambda_{0}$, $d_{z}=d_{r l}=d_{y}=0.5 \lambda_{0}$.

along the E-plane. This is illustrated in Figs. 4 and 5 by varying the array size and the thickness of the coating, respectively.

In Fig. 4, the effect of the array size on the blindness mechanism is investigated. This is achieved by observing the variation in $|R|$ versus scan angle in the E-plane for arrays of $7 \times 7,11$ $\times 11$ and $15 \times 15 \hat{z}$ - and $\hat{\phi}$-directed printed dipoles on a coated cylinder with $a=4 \lambda_{0}$ and $t_{h}=0.06 \lambda_{0}$. As in the previous numerical example, results for planar array are also included for comparison purposes, and $|R|$ values are evaluated for the center elements (which are conjugate matched to broadside scan) of all the arrays. When the size of the array is increased (by adding more elements), surface waves are guided more efficiently along the E-plane for the planar and cylindrical array of $\hat{z}$-directed dipoles. In fact, surfaces waves are stronger for the cylindrical array of $\hat{z}$-directed dipoles when compared to the planar ones [22]. This results in a significant change in the shape of $|R|$ as shown in Fig. 4. Based on these results, scan blindness is not possible for the $7 \times 7$ arrays [see Fig. 4(a)]. However, a peak

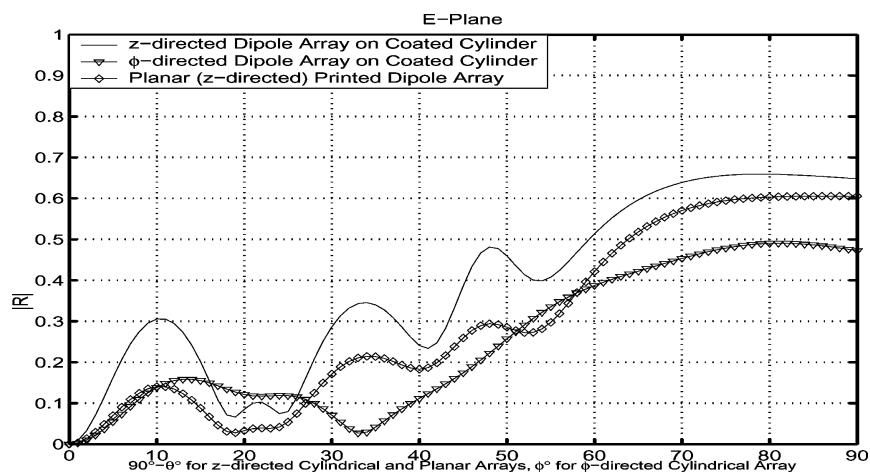

(a)

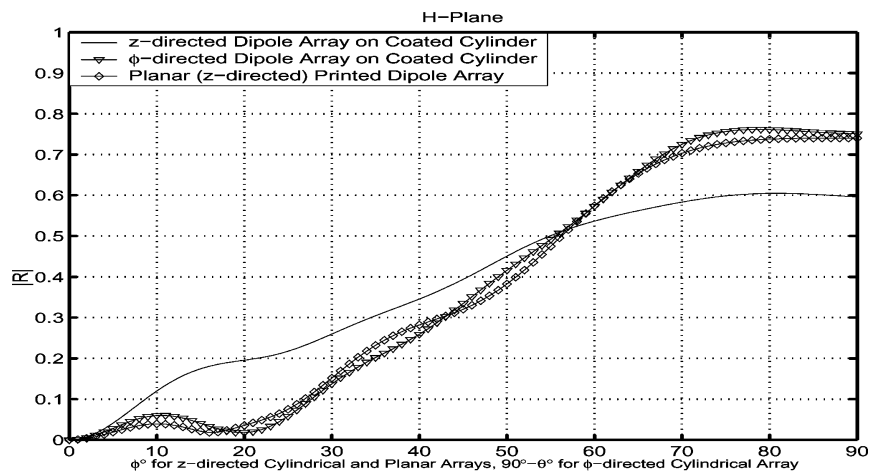

(b)

Fig. 5. Magnitude of the reflection coefficient, $|R|$, of the middle element versus scan angle comparison for $11 \times 11$ cylindrical arrays of $\hat{z}$ - and $\hat{\phi}$-directed printed dipoles, and the same array (of $\hat{z}$-directed dipoles) on a planar grounded dielectric slab along the (a) E-plane and (b) H-plane. Array and host body parameters are: $(L, W)=\left(0.39 \lambda_{0}, 0.01 \lambda_{0}\right), \epsilon_{r}=3.25, t_{h}=0.02 \lambda_{0}$, $d_{z}=d_{r l}=d_{y}=0.5 \lambda_{0}, a=3 \lambda_{0}$.

in the $|R|$ value appears around $\theta=41^{\circ}\left(90^{\circ}-\theta=49^{\circ}\right)$ for both the planar and cylindrical array of $11 \times 11 \hat{z}$-directed printed dipoles [Fig. 4(b)]. This may suggest a potential blindness around this angle even though $|R|<1$. Finally, observing a scan blindness is possible for the cylindrical array of $15 \times$ $15 \hat{z}$-directed dipoles around $\theta=36^{\circ}\left(90^{\circ}-\theta=54^{\circ}\right)$ where $|R| \approx 2.35$ as clearly seen in Fig. 4(c). As expected, the middle element of this array has an impedance with a negative real part around this angle and it delivers power to its generator. For the same sized (i.e., $15 \times 15$ ) planar array, a potential blindness phenomenon also exists around the same angle since $|R|$ is nearly unity. On the other hand, $|R|$ values for the cylindrical array of $\hat{\phi}$-directed dipoles do not change dramatically with the variations in the array size as shown in Fig. 4, and the possibility of scan blindness is not observed. The best way to explain this result is to consider how the curvature of the coated cylinder affects the surface waves for this array. As the surface waves propagate along the E-plane, they continuously shed from the surface due to the curvature. Therefore, along the E-plane ( $\hat{\phi}$-directed dipoles), surface waves are significantly weaker than those of the planar case [23]. Consequently, when the array size is increased, shedding of the waves from the surface continues to be more dominant than the guiding of these waves.

Results given in Fig. 3(a) and (b) are repeated for a thinner coating in Fig. 5 to further emphasize the importance of the surface waves on the blindness mechanism. Parameters used 


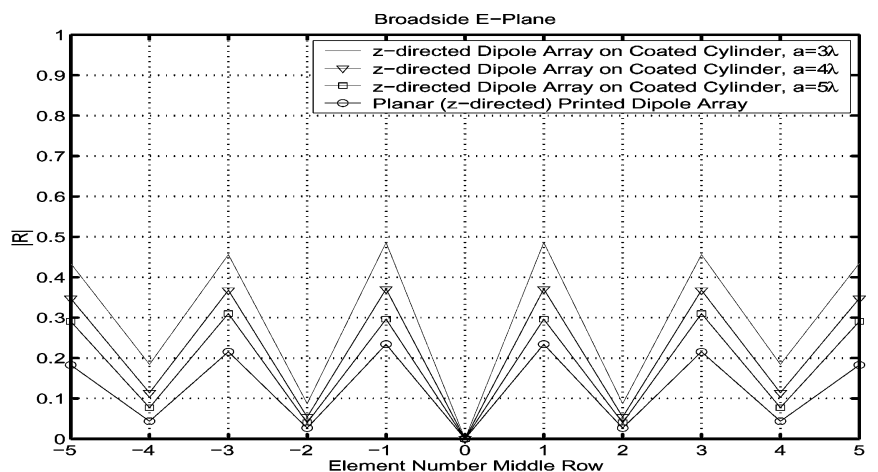

(a)

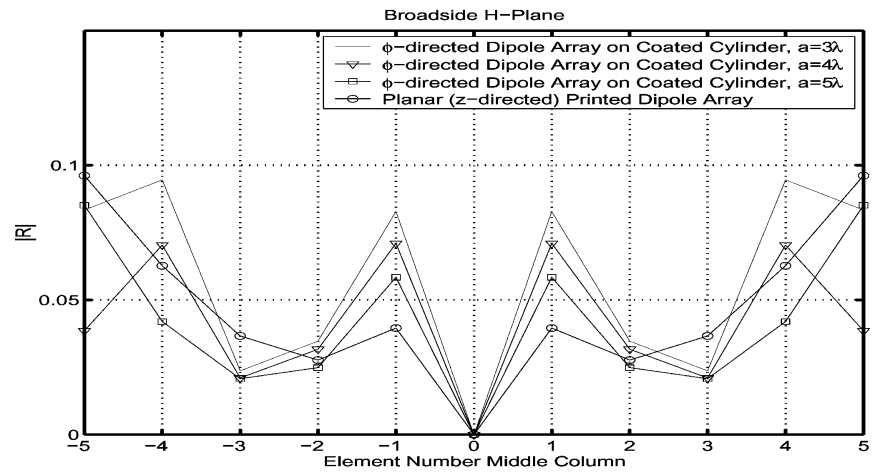

(b)

Fig. 6. (a) $\left|R_{\text {mid }}^{n m}\right|$ versus element position across the E-plane $(\mathrm{n}=-5: 5$, $\mathrm{m}=0)$ of an $11 \times 11$ element $\hat{z}$-directed dipole array on coated cylinders with radii $a=3 \lambda_{0}, a=4 \lambda_{0}, a=5 \lambda_{0}$ and $a=\infty$ (planar), and (b) same as (a) for an $11 \times 11$ element $\hat{\phi}$-directed dipole array across the $\mathrm{H}$-plane $(\mathrm{n}=0$, $\mathrm{m}=-5: 5)$. Other parameters are $(L, W)=\left(0.39 \lambda_{0}, 0.01 \lambda_{0}\right), \epsilon_{r}=3.25$, $t_{h}=0.06 \lambda_{0}, d_{z}=d_{r l}=d_{y}=0.5 \lambda_{0}$.

in Fig. 3 are kept the same except the coating thickness is decreased from $0.06 \lambda_{0}$ to $0.02 \lambda_{0}$. A decrease in the thickness of the coating diminishes the strength of the surface waves, which avoids the possibility of a scan blindness phenomenon in both planes. However, $|R|$ for the cylindrical array of $\hat{z}$-directed dipoles is still higher than that of a planar case, and a small local peak around $\theta=41^{\circ}\left(90^{\circ}-\theta=49^{\circ}\right)$ (which would increase for thicker substrates) is still visible as shown in Fig. 5(a). Note that the effect of the thickness and the relative dielectric constant $\left(\epsilon_{r}\right)$ on scan blindness phenomenon are similar. As it is well known, the "electrical thickness," which depends on the physical thickness, dielectric constant and wavelength, is what matters when surface waves are considered.

The effect of the cylinder radius is discussed next in Fig. 6 by plotting $\left|R_{\text {mid }}^{n m}\right|$ as a function of element position for $11 \times 11$ element arrays, where the definition given in (8) is used. In all cases, broadside scan is considered. In Fig. 6(a), $\left|R_{\text {mid }}^{n m}\right|$ across the E-plane ( $\hat{z}$-direction, i.e., for the elements of the middle row, $\mathrm{n}=-5: 5, \mathrm{~m}=0$ ) of a $\hat{z}$-directed printed dipole array is shown. Similarly in Fig. 6(b), $\left|R_{\text {mid }}^{n m}\right|$ across the H-plane ( $\hat{z}$-direction, i.e., for the elements of the middle column, $n=0$, $\mathrm{m}=-5: 5$ ) of a $\hat{\phi}$-directed printed dipole array is given. As seen from these figures, the input impedance across these finite arrays is nonuniform ([5]), in particular across the E-plane of cylindrical $\hat{z}$-directed dipole arrays. In this plane, such a nonuniformity increases as the size of the radius is decreased, and

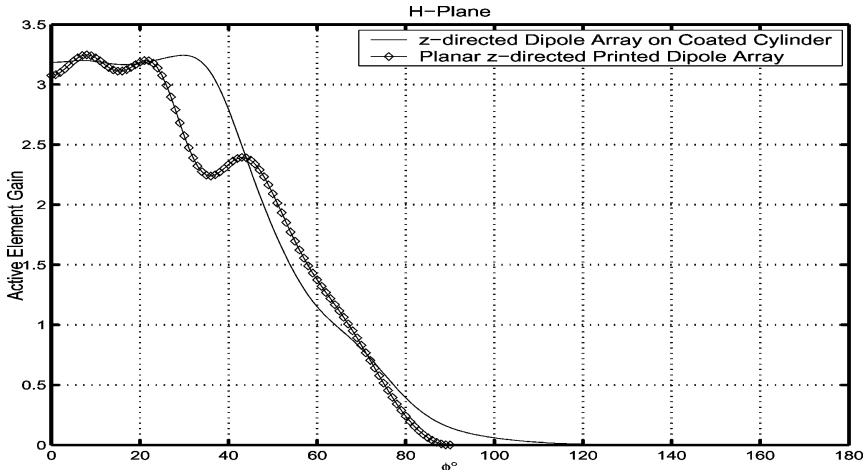

(a)

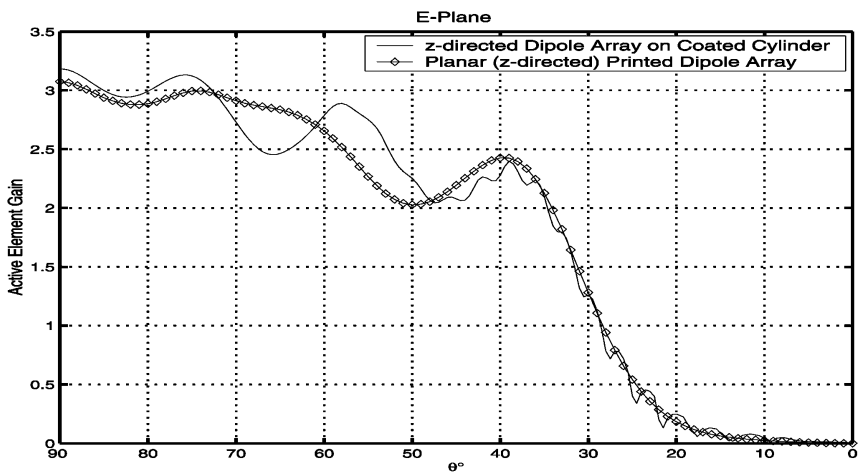

(b)

Fig. 7. (a) H-plane, (b) E-plane active element gain patterns for $15 \times 15 \hat{z}$-directed printed dipoles on a $4 \lambda_{0}$ cylinder and the same array on a planar grounded dielectric slab. Other array and host body parameters are the same as in Fig. 4(c).

relatively high variations in $\left|R_{\text {mid }}^{n m}\right|$ is observed when two consecutive elements are considered. This observation also manifests effects of the surface waves along the axial direction of the coated cylinder. Their strength increases with the decreasing radius [22] [also shown in Fig. 2(a)]. Besides, the variation of $\left|R_{\text {mid }}^{n m}\right|$ is symmetric with respect to the center element in both planes, where the center element is perfectly matched at broadside $\left(\left|R_{\text {mid }}^{\text {mid }}\right|=0\right)$ and others are either slightly or considerably mismatched. Finally, as expected, the results for the cylinder approach that of a planar case as the radius of the cylinder increases.

Fig. 7 compares the finite arrays of printed dipoles on coated cylinders with their planar counterparts using the active element gain patterns defined in (10). Active element gain patterns corresponding to the cylindrical array of $\hat{z}$-directed dipoles discussed in Fig. 4(c) are shown in Fig. 7. These patterns were generated by feeding only the center element of the array and terminating all elements in $Z_{T}=15.3-j * 136.5$, which is the conjugate of the isolated dipole input impedance. First, the $\mathrm{H}$-plane active element gain pattern is shown in Fig. 7(a). Along this plane, scan blindness is not observed since the surface waves are weak especially for the cylindrical case ( $\hat{z}$-directed dipoles). Hence, the gain pattern is very smooth and nearly no oscillations are observed. Note that planar results are valid up to $\phi=90^{\circ}$ due to the infinite substrate and ground plane assumption. On the other hand, for the same arrays, the active element gain pattern is very interesting along the E-plane, where scan blindness was said to 
be possible around $\theta=36^{\circ}\left(90^{\circ}-\theta=54^{\circ}\right)$ for the cylindrical array of $\hat{z}$-directed dipoles based on Fig. 4(c). A null or a dip was expected around this angle in this plane for the cylindrical case. Although the pattern in Fig. 7(b) corresponding to the cylindrical case is more oscillatory than that of the planar one, no null in the pattern is observed. The oscillations in the pattern are due to the surface waves which alter the array current distribution and make it more oscillatory [which can be deduced from the $|R|$ versus element position plots in Fig. 6(a)]. One way to explain this result is to check how many dipoles in the array have a negative resistance (i.e. $\operatorname{Re}\left(Z_{\text {in }}\right)<0$ equivalent to $|R|>1$ ) around this angle. It is observed that if only a small portion of the array elements have a negative resistance, then only a small amount of power is delivered to these elements from the rest of the array elements with $\operatorname{Re}\left(Z_{\text {in }}\right)>0$, and the remaining power is still radiated. Therefore, a potential "scan blindness" may not manifest itself as a visible dip in the gain pattern. In light of this discussion, this cylindrical array of $\hat{z}$-directed dipoles considered in Fig. 4(c), is excited for a scan of $(\theta, \phi)=\left(36^{\circ}, 0^{\circ}\right)$, which corresponds to the "blindness angle" [w.r. to $|R|>1$ result shown in Fig. 4(c)]. The input impedance of all its elements are plotted on the complex impedance plane in Fig. 8(a). The elements experiencing a negative resistance are marked and their locations in the array are shown. Observe that only a small number of elements around the middle of the array have the property $\operatorname{Re}\left(Z_{\text {in }}\right)<0$ and they extract little power from the array. If more elements had negative resistance, then blindness will be observed in the gain patterns in the form of a visible dip. Finally, in an infinite array, which can be considered as the limiting case, the input impedance of all elements are identical and purely imaginary at the blindness angle. Therefore, a complete blindness would occur and manifests itself as a null in the gain pattern in this plane.

A similar investigation is also performed for the cylindrical array of $\hat{\phi}$-directed dipoles. They are excited at a scan of $(\theta, \phi)=\left(90^{\circ}, 54^{\circ}\right)$ such that the E-plane scan is performed exactly the same as $\hat{z}$-directed dipole array case. It is observed that $\operatorname{Re}\left(Z_{\text {in }}\right)$ values for all elements in this case are positive as clearly seen in Fig. 8(b). Based on this information and considering all the previously given numerical results, we can conclude that array element orientation with respect to the curvature of the supporting structure plays a significant role. Considerably different behaviors are observed concerning scan blindness phenomenon for finite arrays of axially and circumferentially directed printed dipoles on cylindrical platforms as well as their planar counterparts.

Finally, the normalized far-field radiation patterns pertaining to $13 \times 13$ arrays of $\hat{z}$ - and $\hat{\phi}$-directed dipoles on coated cylinders with radii $3 \lambda_{0}$ and $5 \lambda_{0}$, and their comparison with patterns of a planar array are shown in Fig. 9. The thickness of the coating is $0.06 \lambda_{0}$ for all cases. Fig. 9(a) shows the E-plane pattern for the cylindrical array of $\hat{z}$-directed dipoles. In this plane, effects of the curvature on the radiation pattern is minimum. Hence, as expected, patterns resemble to the planar case. However, along the H-plane, where the curvature affects the most, patterns are quite different as seen in Fig. 9(b). Agreement with the planar case is observed only in the main beam as well as in the first sidelobe levels. For the cylindrical array of $\hat{\phi}$-directed dipoles, the curva-

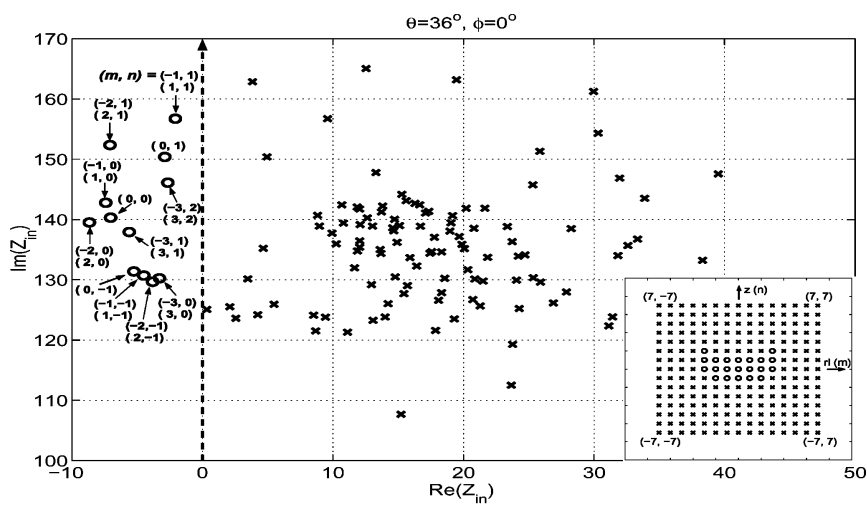

(a)

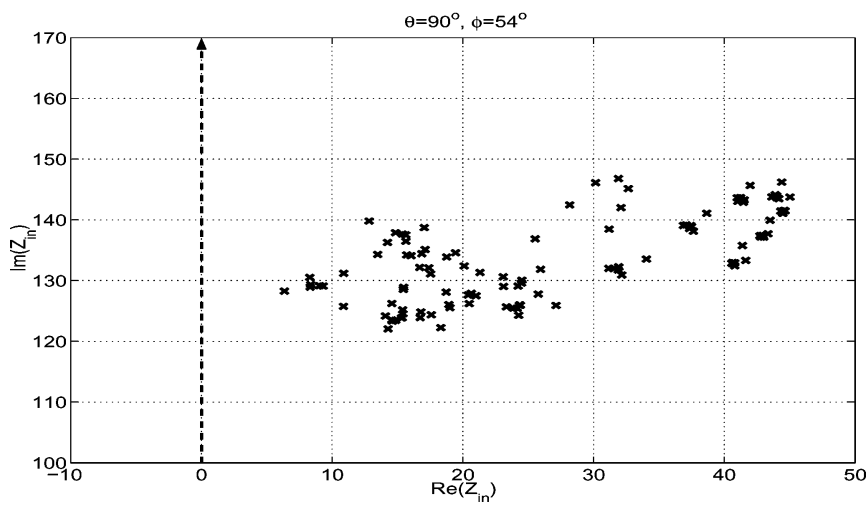

(b)

Fig. 8. (a) Input impedance $\left(Z_{\text {in }}\right)$ of all elements for a $15 \times 15 \hat{z}$-directed dipoles on a $4 \lambda_{0}$ cylinder on the complex impedance plane. Location of the dipoles in the array with negative real resistance values are marked with "o" (rest is marked with "x"). (b) Same as (a) for the same sized $\hat{\phi}$-directed printed dipole array on the same cylinder. Other array and host body parameters are the same as in Fig. 4(c).

ture plays a very significant role along the E-plane. This result is expected since the array elements are oriented perpendicular to the axis of the cylinder. Thus, other than the main beam, a complete disagreement with the planar case is expected and observed as shown in Fig. 9(c). The H-plane patterns are shown in Fig. 9(d) where the curvature does not have a significant impact and a good agreement is observed with the planar results. In the evaluation of all patterns, all dipoles are excited uniformly and no special beam forming technique is applied in the excitation of the arrays. Note that the ground plane and the substrate are assumed to be infinite for the planar case and the dipoles are $\hat{z}$-directed. Also cylinders are assumed to be infintely long along the $z$-direction (parallel to axis of cylinder). Therefore, patterns for planar array as well as the E-plane pattern for the cylindrical array of $\hat{z}$-directed dipoles and $\mathrm{H}$-plane pattern for the cylindrical array of $\hat{\phi}$-directed dipoles are evaluated from $-90^{\circ}$ to $90^{\circ}$.

\section{CONCLUSION}

In this paper, a rigorous investigation of surface waves and their effect on scan blindness phenomenon for conformal finite phased arrays of printed dipoles has been performed. Furthermore, effects of several array and supporting structure parameters on the basic performance metrics of arrays and on the 


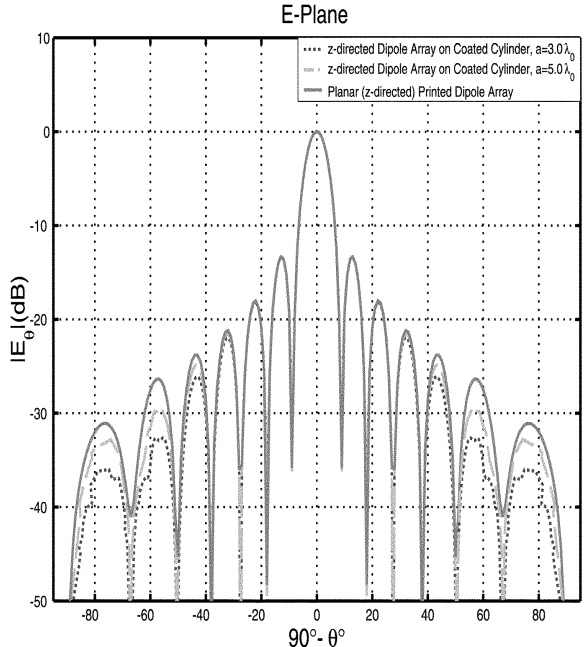

(a)

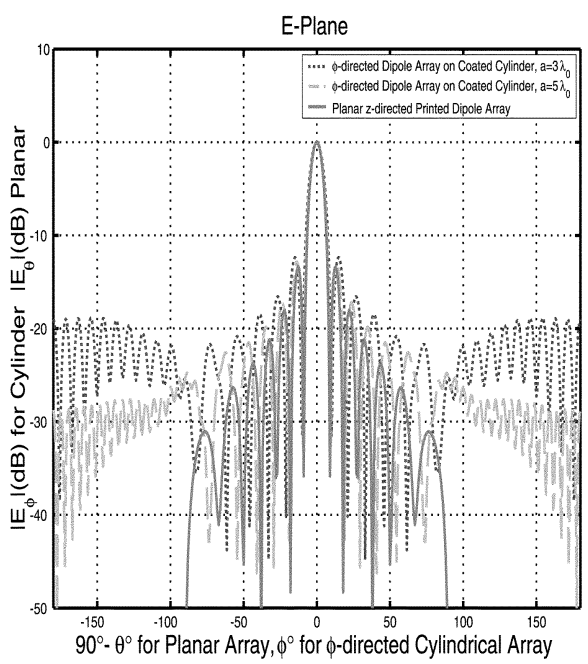

(c)

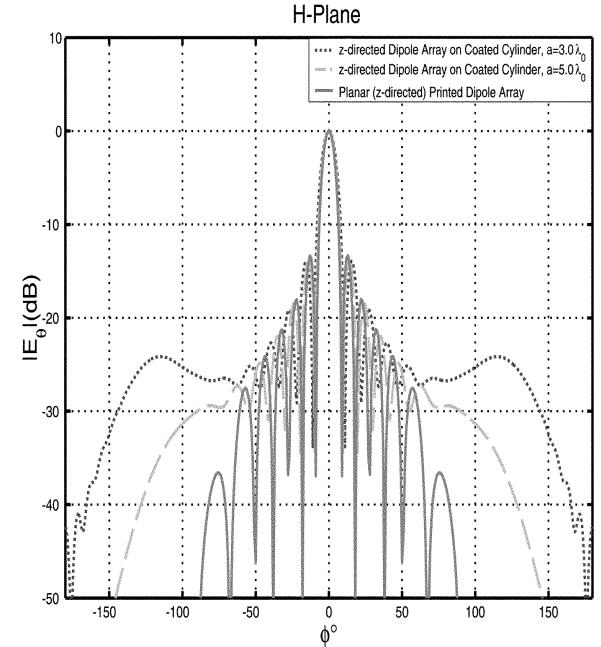

(b)

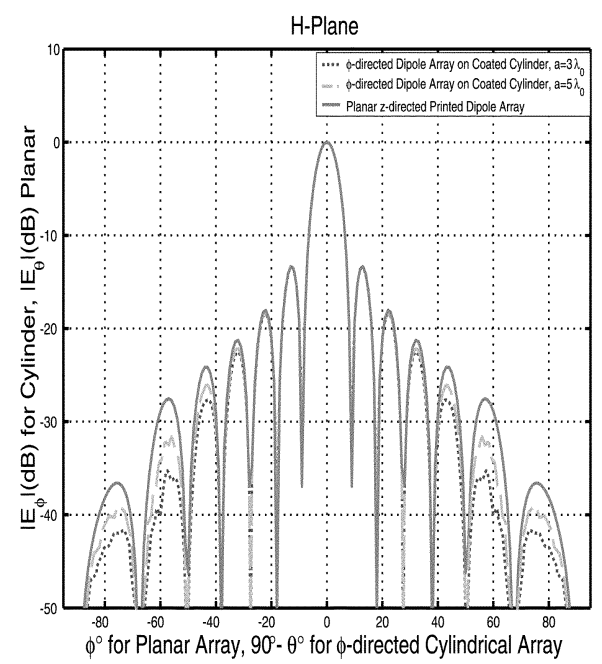

(d)

Fig. 9. Far-field patterns of $13 \times 13$ printed dipole arrays on $3 \lambda_{0}, 5 \lambda_{0}$ cylinders and on planar substrates. Patterns for planar and cylindrical $\hat{z}$-directed dipole arrays along the (a) E-plane and (b) H-plane. Patterns for planar and cylindrical $\hat{\phi}$-directed dipole arrays along the (c) E-plane and (d) H-plane. All arrays are phased to radiate along the broadside direction. Other array and host body parameters are: $(L, W)=\left(0.39 \lambda_{0}, 0.01 \lambda_{0}\right), \epsilon_{r}=3.25, t_{h}=0.06 \lambda_{0}, d_{z}=d_{r l}=$ $d_{y}=0.5 \lambda_{0}$.

blindness mechanism have been discussed. To be able to address these issues, a computationally optimized and very accurate hybrid full wave analysis method has been used. This hybrid method is based on the combination of the MoM with three high frequency based asymptotic Green's function representations of an appropriate Green's function in the spatial domain. Several relatively large but finite arrays pertaining to both axially and circumferentially oriented printed dipoles on coated cylinders with different radii have been studied.

In addition to standard parameters (size of the array, thickness of the substrate, value of the dielectric constant, etc.) that affect the blindness mechanism in finite phased arrays of printed dipoles on planar grounded slabs, it is shown here that the curvature of the supporting structure and the orientation of the array elements significantly alter the surface waves excited within the substrate and in turn the blindness mechanism. Consequently, i) finite phased arrays of printed dipoles on coated cylinders and similar arrays on planar grounded slabs show different behavior in terms of scan blindness, and ii) unlike planar arrays where scan blindness is mainly governed by the array related factors (substrate parameters, element spacings, etc.) rather than the particular element orientation, scan blindness in cylindrical arrays of printed dipoles is also governed by the orientation of the array elements with respect to the supporting structure. Under the same excitations and with the same array and host body parameters, axially oriented printed dipole arrays can exhibit scan blindness phenomenon, but it may not occur for arrays of circumferentially oriented printed dipoles.

\section{REFERENCES}

[1] R. J. Mailloux, Phased Array Antenna Handbook. Boston, MA: Artech House, 1994.

[2] R. C. Hansen, Phased Array Antennas. New York: Wiley, 1998.

[3] D. M. Pozar and D. H. Schaubert, "Scan blindness in infinite phased arrays of printed dipoles," IEEE Trans. Antennas Propag., vol. 32, no. 6, pp. 602-610, Jun. 1984.

[4] - "Analysis of an infinite array of rectangular microstrip patches with idealized probe feeds," IEEE Trans. Antennas Propag., vol. 32, no. 10, pp. 1101-1107, Oct 1984.

[5] D. M. Pozar, "Analysis of finite phased arrays of printed dipoles," IEEE Trans. Antennas Propag., vol. 33, no. 10, pp. 1045-1053, Oct 1985. 
[6] _ , "Finite phased arrays of rectangular microstrip patches," IEEE Trans. Antennas Propag., vol. 34, no. 5, pp. 658-665, May 1986.

[7] A. K. Skrivervik and J. R. Mosig, "Finite phased arrays of microstrip patch antennas: The infinite array approach," IEEE Trans. Antennas Propag., vol. 40, no. 5, pp. 579-582, May 1992.

[8] O. A. Civi, V. B. Ertürk, P. H. Pathak, P. Janpugdee, and H. T. Chou, "A hybrid UTD-MoM approach for the efficient analysis of radiation/ scattering from large, printed finite phased arrays," in IEEE APS Int. Symp. URSI Radio Science Meeting, Boston, MA, Jul. 2001, vol. 2, pp. 806-809.

[9] A. Polemi, A. Toccafondi, and S. Maci, "High-frequency Green's function for a semi-infinite array of electric dipoles on a grounded slab-Part 1: Formulation," IEEE Trans. Antennas Propag., vol. 49, no. 12 , pp. $1667-1677$, Dec. 2001

[10] H. T. Chou, H. K. Ho, P. H. Pathak, P. Nepa, and O. A. Civi, "Efficient hybrid discrete Fourier transform-moment method for fast analysis of large rectangular arrays," Proc. Inst. Elect. Eng. Microwave Antennas Propagat., vol. 149, pp. 1-6, 2002.

[11] V. B. Ertürk and H. T. Chou, "Efficient analysis of large phased arrays using iterative MoM with DFT-based acceleration algorithm," Microwave Opt. Technol. Lett., vol. 39, pp. 89-94, Oct. 2003.

[12] D. H. Schaubert, J. A. Aas, M. E. Cooley, and N. E. Buris, "Moment method analysis of infinite stripline-fed tapered slot antenna arrays with a ground plane," IEEE Trans. Antennas Propag., vol. 42, no. 8, pp. 1161-1166, Aug. 1994.

[13] D. M. Pozar, "Scanning characteristics of infinite arrays of printed antenna subarrays," IEEE Trans. Antennas Propag., vol. 40, pp. 666-674, Jun. 1992.

[14] _ " "General relations for a phased array of printed antennas derived from infinite current sheets," IEEE Trans. Antennas Propag., vol. 33, no. 5, pp. 498-504, May 1985.

[15] — , "Performance of an infinite array of monopoles in a grounded dielectric slab," Proc. Inst. Elect. Eng. Microwave Antennas Propagat., vol. 137, pp. 117-120, Apr. 1990.

[16] J. P. R. Bayard, M. E. Cooley, and D. H. Schaubert, "Analysis of infinite arrays of printed dipoles on dielectric sheets perpendicular to a ground plane," IEEE Trans. Antennas Propag., vol. 39, no. 12, pp. 1722-1732, Dec. 1991.

[17] D. H. Schaubert, "A class of E-plane scan blindnesses in single-polarized arrays of tapered-slot antennas with a ground plane," IEEE Trans. Antennas Propag., vol. 44, no. 7, pp. 954-959, Jul. 1996.

[18] M. Davidovitz, "Extension of the E-plane scanning range in large microstrip arrays by substrate modification," IEEE Microwave Guided Wave Lett., vol. 2, pp. 492-494, Dec. 1992.

[19] R. B. Waterhouse and N. V. Shuley, "Scan performance of infinite arrays of microstrip patch elements loaded with varactor diodes," IEEE Trans. Antennas Propag., vol. 41, no. 9, pp. 1273-1280, Sep. 1993.

[20] R. B. Waterhouse, "The use of shorting posts to improve the scanning range of probe-fed microstrip patch phased arrays," IEEE Trans. Antennas Propag., vol. 44, no. 3, pp. 302-309, Mar. 1996.

[21] V. B. Ertürk and R. G. Rojas, "Efficient analysis of input impedance and mutual coupling of microstrip antennas mounted on large coated cylinders," IEEE Trans. Antennas Propag., vol. 51, no. 4, pp. 739-749, Apr. 2003.

[22] V. B. Ertürk, K. W. Lee, and R. G. Rojas, "Analysis of finite arrays of axially directed printed dipoles on electrically large cylinders," IEEE Trans. Antennas Propag., vol. 52, no. 10, pp. 2586-2595, Oct. 2004.

[23] V. B. Ertürk and B. Güner, "Analysis of finite arrays of circumferentially oriented printed dipoles on electrically large cylinders," $M i$ crowave Opt. Technol. Lett., vol. 42, pp. 299-304, Aug. 2004.

[24] S. Barkeshli, P. H. Pathak, and M. Marin, "An asymptotic closed-form microstrip surface Green's function for the efficient moment method analysis of mutual coupling in microstrip antennas," IEEE Trans. Antennas Propag., vol. 38, no. 9, pp. 1374-1383, Sep. 1990.

[25] V. B. Ertürk and R. G. Rojas, "Efficient computation of surface fields excited on a dielectric coated circular cylinder," IEEE Trans. Antennas Propag., vol. 48, no. 10, pp. 1507-1516, Oct. 2000.

[26] _ "Paraxial space-domain formulation for surface fields on dielectric coated circular cylinder," IEEE Trans. Antennas Propag., vol. 50, no. 11 , pp. 1577-1587, Nov. 2002.

[27] V. B. Ertürk, "Efficient hybrid MoM/Green's function technique to analyze conformal microstrip antennas and arrays," Ph.D. dissertation, Dept. Electrical Eng., The Ohio State Univ., Columbus, 2000.

[28] J. Ashkenazy, S. Shtrikman, and D. Treves, "Electric surface current model for the analysis of microstrip antennas on cylindrical bodies," IEEE Trans. Antennas Propag., vol. 33, no. 3, pp. 295-300, Mar. 1985.

[29] R. A. Martin and D. H. Werner, "A reciprocity approach for calculating the far-field radiation patterns of a center-fed helical microstrip antenna mounted on a dielectric coated circular cylinder," IEEE Trans. Antennas Propag., vol. 49, no. 12, pp. 1754-1762, Dec. 2001.
[30] P. D. Patel, "Approximate location of scan-blindness angle in printed phased arrays," IEEE Antennas Propag. Mag., vol. 34, pp. 53-54, Oct. 1992.

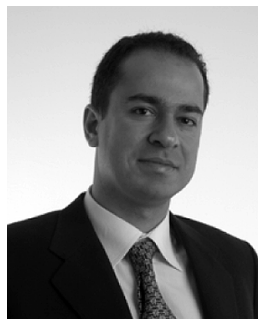

Vakur B. Ertürk (M'00) received the B.S. degree in electrical engineering from the Middle East Technical University, Ankara, Turkey, in 1993, and the M.S. and Ph.D. degrees from The Ohio-State University (OSU), Columbus, in 1996 and 2000, respectively.

He is currently an Assistant Professor with the Electrical and Electronics Engineering Department, Bilkent University, Ankara. His research interests include the analysis and design of planar and conformal arrays, active integrated antennas, scattering from and propagation over large terrain profiles as well as metamaterials.

Dr. Ertürk has served as the Secretary/Treasurer of IEEE Turkey Section as well as the Turkey Chapter of the IEEE Antennas and Propagation, Microwave Theory and Techniques, Electron Devices and Electromagnetic Compatibility Societies.

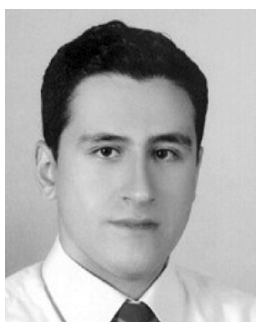

Onur Bakır (S'05) was born in Kayseri, Turkey, in 1980. He received his B.S. degree in electrical engineering from Bilkent University, Bilkent, Turkey, in 2003.

He is currently working toward his M.S. degree at Bilkent University where he has been a Research Assistant since 2003. His research interests include design and analysis of microstrip antennas on planar and curved surfaces and computational electromagnetics.

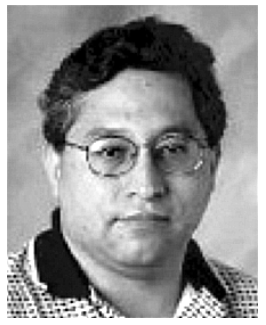

Roberto G. Rojas (S'80-M'85-SM'90-F'01) received the B.S.E.E. degree from New Mexico State University, Las Cruces, in 1979 and the M.S. and $\mathrm{Ph} . \mathrm{D}$. degrees in electrical engineering from The Ohio State University (OSU) Columbus, in 1981 and 1985 , respectively

$\mathrm{He}$ is currently a Professor in the Department if Electrical and Computer Engineering with The Ohio State University. His current research interests include the analysis and design of conformal arrays, active integrated arrays, nonlinear microwave circuits, as well as the analysis of electromagnetic radiation and scattering phenomena in complex environments.

Dr. Rojas is an elected Member of the International Scientific Radio Union (URSI), Commission B. He won the 1988 R.W.P. King Prize Paper Award, the 1990 Browder J. Thompson Memorial Prize Award, both given by IEEE, the 1989 and 1993 Lumley Research Awards, given by the College of Engineering at The Ohio State University. He has served as Chairman, Vice-Chairman and Secretary/Treasurer of the Columbus, $\mathrm{OH}$, chapter of the IEEE Antennas and Propagation and Microwave Theory and Techniques Societies.

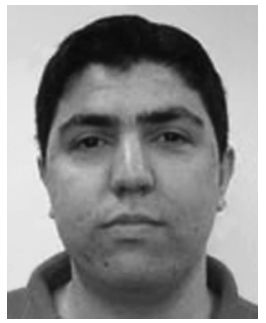

Baris Güner received the B.S. and M.S. degrees in electrical and electronics engineering from the Bilkent University, Ankara, Turkey, in 2002 and 2004, respectively.

$\mathrm{He}$ is currently a Graduate Research Associate with the Department of Electrical and Computer Engineering, The Ohio State University, (OSU), Columbus. His current research interests are in microwave remote sensing. 